\title{
Creating Accessible Web Pages in Dreamweaver
}

\section{Judi Franz University of California, Irvine}

Note: This tutorial was created using Dreamweaver MX2004. A new version (Dreamweaver 8) has just been released in September, 2005. I assume the settings will be similar, but have not yet had a chance to use the newer version.

Creating truly accessible web pages can be a daunting undertaking, especially if you want your site to include graphics and interactive elements. However, you can make a good start at accessibility by following some basic guidelines when adding images, tables, and other elements to your pages. Macromedia's Dreamweaver makes creating accessible web pages easier for those without knowledge of HTML. After changing a few settings in the Preferences, Dreamweaver will prompt the user for necessary information when adding certain elements to a web page. After you have launched the program, select Dreamweaver (8) Preferences (Mac) or Edit (8) Preferences (Windows).

Dreamweaver frle Edit Vlew Insert Mor
About Macromedla Dreamweaver MX 2004
Keyboard Shortcuts...
Preferences..
Services
Hide Dreamweaver
Hide Others
Show All
Quit Dreamweaver

When the Preferences panel opens, select Accessibility under the Category listing along the left side of the window.

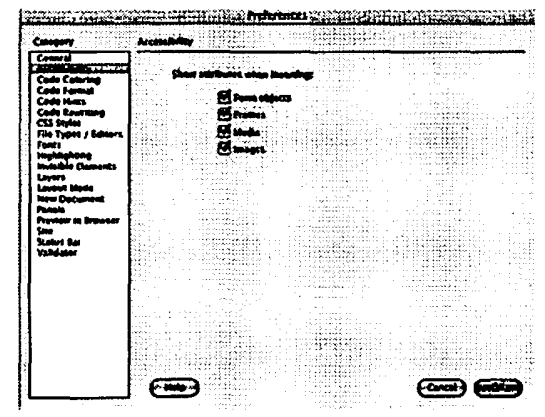

Check all four boxes (Form objects, Frames, Media, Images), and click $\mathbf{O K}$. Now, open a new document (File (8) New (8) Basic Page / HTML). 
Images

Tables
Since the most frequently used elements are images and tables, this tutorial will cover these. Once you are used to following Dreamweaver's prompts for accessibility information, you should be able to work with form elements, frames and media as well. Often, the main thing to remember is naming each element on the page appropriately.

From the Insert menu, select Image. You will be prompted to enter Alternate text and/or a URL pointing to a long description of the image. Alternate text will be read aloud by a visually-impaired user's screen reading software. It should be descriptive, but not too long. The alternate text will also appear when any user hovers their mouse over the image.

For most images, which are used for aesthetic purposes only, briefly descriptive alternate text is usually all that is needed. If the image serves a larger purpose (for example, it illustrates some aspect of the culture the students are expected to comment on or use in some way), then you would want to link to a separate page with a longer, much more descriptive, explanation of its contents.

Alternate text can also be added in the Properties panel when an image is selected on the page. This is helpful if you are trying to update a page created earlier.

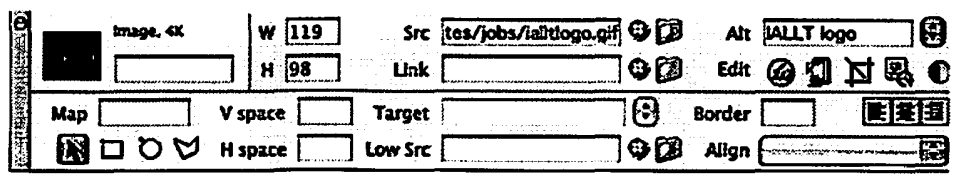

Tables come in two different types in web pages: layout tables and data tables. Layout tables are often used to set up the overall look of a page, and to keep all of the individual elements in place so that the page looks similare in different browsers. In Dreamweaver, layout tables are created in Layout Mode, and this process inserts a comment in the HTML code identifying it as a layout table. 


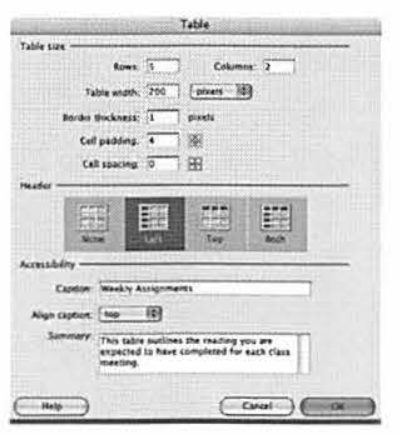

Weekly Assignments

\begin{tabular}{|c|l|}
\hline Week & Reading \\
\hline 1 & Chapter I \\
\hline 2 & Chapter 2 \\
\hline 3 & Chapters 3-4 \\
\hline 4 & Chapters 5-6 \\
\hline
\end{tabular}

Here is the resultant table from the prompts in the previous image:

Accessibility is highest when the table can be read in a linearized manner. Designating a header row also helps screen readers make sense of the information presented for visually impaired users. 


\section{Franz}

